BULL. AUSTRAL. MATH. SOC.

VOL. 17 (1977), 423-432.

\title{
On the stability of impulsively perturbed differential systems
}

\section{S.G. Pandit}

This paper deals with the study of uniform asymptotic stability of the measure differential system $D x=F(t, x)+G(t, x) D u$, where the symbol $D$ stands for the derivative in the sense of distributions. The system is viewed as a perturbed system of the ordinary differential system $x^{\prime}=F(t, x)$, where the perturbation term $G(t, x) D u$ is impulsive and the state of the system changes suddenly at the points of discontinuity of $u$. It is shown, under certain conditions, that the uniform asymptotic stability property of the unperturbed system is shared by the perturbed system. To do this, the well-known Gronwall integral inequality is generalized so as to be applicable to LebesgueStieltjes integrals.

\section{Introduction}

For any vector $x=\left(x_{1}, \ldots, x_{n}\right) \in R^{n}$, let

$$
|x|=\sum_{i=1}^{n}\left|x_{i}\right|
$$

Denote by $C\left[X, R^{n}\right]$, the class of all continuous mappings from $X$ into $R^{n}$. Let $J=\left[t_{0}, \infty\right), t_{0} \geq 0$. We consider the following measure differential equation [4]:

Received 7 July 1977. The author thanks Professors V. Raghavendra and S.G. Deo for their kind help while he was preparing this paper. 


$$
D x=F(t, x)+G(t, x) D u,
$$

where $x \in R^{n}$, Du denotes the distributional derivative of the function $u \quad F, G \in C\left[J \times R^{n}, R^{n}\right]$, and $u: J \rightarrow R$ is a right-continuous function of bounded variation on every compact subinterval of $J$. Here $D u$ can be identified with a Stieltjes measure and has the effect of instantaneously changing the state of the system at the points of discontinuity of $u$. Equation (1.1) arises in several ways. For example, when $F(t, x)=A x$, $G(t, x)=1$, and $u(t)=u_{1}(t)+\sum_{k=1}^{\infty} a_{k} \delta\left(t-t_{k}\right)$, where $A$ is an $n \times n$ constant matrix, $u_{1}(t)$ is a locally integrable function, $a_{k} \in R^{n}$, and $\delta\left(t-t_{k}\right)$ is the Dirac function, system (1.I) may be regarded as a link in an automatic control chain transmitting the distribution associated with the function $u$. System (1.1) may also be treated as a perturbed system of the ordinary differential system

$$
x^{\prime}=F(t, x) \quad(1=d / d t),
$$

where the perturbation $G(t, x) D u$ is impulsive [4, 6]. In this latter set up, it is natural to ask: under what conditions are the stability properties of (1.2) shared by the solutions of (1.1)? As pointed out in $[4,6]$, it is difficult to get a satisfactory answer to this question. It may be so because differential and integral inequalities play a crucial role in the stability theory. But the fact that the solutions of (1.1) are discontinuous renders the existing differential inequalities unapplicable, and the integral inequalities for stieltjes integrals are not available.

The purpose of this paper is to obtain an integral inequality of Gronwall type involving Lebesgue-Stieltjes integrals and apply it to investigate a stability property of solutions of (1.1) with respect to the solutions of (1.2).

We use the following lemmas in our subsequent discussion.

LEMMA 1.1 [3, p. 19]. Let $x$ and $K$ be scalar nonnegative functions defined and integrable on $[a, b]$. Then, for any nonnegative constant $c$, the inequality 


$$
x(t) \leq c+\int_{a}^{t} K(s) x(s) d s, \quad t \in[a, b],
$$

implies

$$
x(t) \leq c \cdot \exp \left(\int_{a}^{t} K(s) d s\right), \quad t \in[a, b]
$$

LEMMA 1.2. Let $(X, M, \mu)$ be a finite measure space and $\left\{E_{n}\right\}$ be a sequence in $M$ such that $E_{1} \supset E_{2} \supset \ldots$ Then $\lim _{n \rightarrow \infty} \mu\left(E_{n}\right)=\mu\left(\prod_{n=1}^{\infty} E_{n}\right)$.

Let $x(t)=x\left(t, t_{0}, x_{0}\right)$ and $y(t)=y\left(t, t_{0}, x_{0}\right)$ be solutions of (1.2) and (1.1) respectively, through $\left(t_{0}, x_{0}\right)$ existing on the right of $t_{0} \geq 0$ in $s(r)$, where $s(r)=\left\{x \in R^{n}:|x| \leq r\right\}$.

DEFINITION [3, p. 51]. The null solution of (1.1) is said to be uniformly asymptotically stable if the following two conditions hold:

(i) for each $\varepsilon>0$, there is a $\delta=\delta(\varepsilon)>0$ such that if $\left|y\left(t^{\prime}\right)\right|<\delta$ for some $t^{\prime} \geq t_{0}$ then $|y(t)|<\varepsilon$ for all $t \geq t^{\prime}$

(ii) there is a $\delta_{0}>0$, and, for each $\varepsilon>0$, a corresponding $T=T(\varepsilon)>0$ such that if $\left|y\left(t^{\prime}\right)\right|<\delta_{0}$ for some $t^{\prime} \geq t_{0}$ then $|y(t)|<\varepsilon$ for all $t>t^{\prime}+T$.

\section{Main theorem}

Since $u$ is of bounded variation on $J$, its discontinuities may be enumerated as $t_{1}<t_{2}<\ldots \cdot u$ has a canonical representation $u=u_{1}+u_{2}$ where $u_{1}$ is an absolutely continuous function of bounded variation and $u_{2}$ is a sum of jump functions, the jumps being those of $u$. It follows that $u^{\prime}$ exists and is equal to $u_{1}^{\prime}$ almost everywhere on $J$. Let $\lambda_{k}=u\left(t_{k}\right)-u\left(t_{k}-\right)$ denote the gump of $u$ at $t=t_{k}$, $k=1,2, \ldots$. In the following, all functions of one variable will be assumed to be defined, real-valued, and measurable on $J$. We call such a 
function $w(t)$ locally du-integrable on $J$ if, for each $t \in J$, its Lebesgue-Stieltjes integral $\int_{t_{0}}^{t} w(s) d u(s)$ is finite.

\section{THEOREM 2.1. Suppose that}

(i) $x$ and $f$ are non-negative functions, locally $d u-$ integrable on $J$;

(ii) $u$ is a right-continuous function of bounded variation on $J$ and $u_{1}^{\prime}$ is non-negative and integrable on $J$;

(iii) the discontinuities $t_{k}, k=1,2, \ldots$, of $u$ are isolated and are such that

$$
\lambda_{k} f\left(t_{k}\right)<1
$$

for each $k \geq 1$; the series

$$
\sum_{k=1}^{\infty} \lambda_{k} f\left(t_{k}\right)
$$

converges absolutely.

Then for any positive constant $c$, the inequality

$$
x(t) \leq c+\int_{t_{0}}^{t} f(s) x(s) d u(s), \quad t \in J,
$$

implies

$$
x(t) \leq P^{-1} \cdot c \cdot \exp \left(\int_{t_{0}}^{t} f(s) u_{1}^{\prime}(s) d s\right), \quad t \in J,
$$

where

$$
P=\prod_{k=1}^{\infty}\left\{1-\lambda_{k} f\left(t_{k}\right)\right\}
$$

Proof. Denote the quantity on the right side of (2.3) by $r(t)$. Then, for $t=t_{1}$, we have 


$$
\begin{aligned}
r\left(t_{1}\right) & =c+\int_{t_{0}}^{t_{1}} f(s) x(s) d u(s) \\
& =c+\int_{t_{0}}^{t_{1}-\varepsilon} f(s) x(s) d u(s)+\int_{t_{1}-\varepsilon}^{t_{1}} f(s) x(s) d u(s),
\end{aligned}
$$

where $\varepsilon>0$ is arbitrary. Since $u$ is absolutely continuous on $\left[t_{0}, t_{1}-\varepsilon\right]$, the discontinuities being isolated, by Lemma 1.1 we obtain

$$
r\left(t_{1}\right) \leq c \cdot \exp \left(\int_{t_{0}}^{t_{1}} f(s) u_{1}^{\prime}(s) d s\right)+\int_{t_{1}-\varepsilon}^{t_{1}} f(s) x(s) d u(s)
$$

We prove that

$$
\int_{t_{1}-\varepsilon}^{t_{1}} f(s) x(s) d u(s)=\lambda_{1} f\left(t_{1}\right) x\left(t_{1}\right)
$$

Consider the measure $\mu$ defined by

$$
\mu(E)=\int_{E} f(s) x(s) d u(s)
$$

Let $\left\{\alpha_{n}\right\}$ be a decreasing sequence of positive real numbers tending to zero and $E_{n}=\left[t_{1}-\alpha_{n}, t_{1}\right]$. Then $E_{1} \supset E_{2} \supset \ldots$ and $\prod_{n=1}^{\infty} E_{n}=\left\{t_{1}\right\}$. By Lemma 1.2, $\mu\left(E_{n}\right) \rightarrow \mu\left(\left\{t_{1}\right\}\right)=\lambda_{1} f\left(t_{1}\right) x\left(t_{1}\right)$. Thus (2.7) is established. Letting $\varepsilon \rightarrow 0_{+}$in (2.6) and using (2.1) and the fact that $x\left(t_{1}\right) \leq r\left(t_{1}\right)$, we obtain

$$
r\left(t_{1}\right) \leq P_{1}^{-1} \cdot c \cdot \exp \left(\int_{t_{0}}^{t_{1}} f(s) u_{1}^{\prime}(s) d s\right)
$$

where

$$
P_{k}=\prod_{n=1}^{k}\left\{1-\lambda_{n} f\left(t_{n}\right)\right\}
$$

Assume 


$$
r\left(t_{m}\right) \leq P_{m}^{-1} \cdot c \cdot \exp \left(\int_{t_{0}}^{t_{m}} f(s) u_{1}^{\prime}(s) d s\right)
$$

for some integer $m \geq 1$. Then

$$
r\left(t_{m+1}\right)=r\left(t_{m}\right)+\int_{\left[t_{m}, t_{m+1}\right]} f(s) x(s) d u(s)
$$

which, as before, gives

$$
\begin{aligned}
r\left(t_{m+1}\right) & \leq r\left(t_{m}\right)\left\{1-\lambda_{m+1} f\left(t_{m+1}\right)\right\}^{-1} \exp \left\{\int_{t_{m}}^{t_{m+1}} f(s) u_{1}^{\prime}(s) d s\right) \\
& \leq P_{m+1}^{-1} \cdot c \cdot \exp \left(\int_{t_{0}}^{t_{m+1}} f(s) u_{1}^{\prime}(s) d s\right) .
\end{aligned}
$$

Therefore, by induction, it follows that

(2.8) $x\left(t_{k}\right) \leq r\left(t_{k}\right) \leq P_{k}^{-1} \cdot c \cdot \exp \left(\int_{t_{0}}^{t_{k}} f(s) u_{l}^{\prime}(s) d s\right), k=1,2, \ldots$

By hypothesis ( $i i i)$, we observe that $P_{k+1} \leq P_{k}$ for each $k$ and that $\lim _{k \rightarrow \infty} P_{k}=P$. Hence (2.8) can be written as

$$
x\left(t_{k}\right) \leq P^{-1} \cdot c \cdot \exp \left(\int_{t_{0}}^{t_{k}} f(s) u_{1}^{\prime}(s) d s\right), k=1,2, \ldots,
$$

so that $(2.4)$ is verified at all points of discontinuity of $u$. Finally, if $t$ is any point of continuity of $u$, then $t \in\left(t_{k-1}, t_{k}\right)$ for some $k \geq 1$. In this case, we have

$$
x(t) \leq r\left(t_{k-1}\right)+\int_{\left(t_{k-1}, t\right]} f(s) x(s) d u(s) .
$$

Since $u$ is absolutely continuous on $\left(t_{k-1}, t\right]$, we may apply Lemma 1.1 along with (2.9) to obtain the desired conclusion. This completes the proof. 
COROLLARY 2.1. Under the conditions of Theorem 2.1, if $g$ is a non-negative function defined and integrable on $J$, then the inequality

$$
x(t) \leq c+\int_{t_{0}}^{t} g(s) x(s) d s+\int_{t_{0}}^{t} f(s) x(s) d u(s), t \in J,
$$

implies

$$
x(t) \leq P^{-1} \cdot c \cdot \exp \left(\int_{t_{0}}^{t}\left\{g(s)+f(s) u_{1}^{\prime}(s)\right\} d s\right\}, \quad t \in J
$$

REMARK 2.1. If $u(t)=u_{1}(t)=t$, then $\lambda_{k}=0$ for each $k$, so that $p=1$ and Theorem 2.1 reduces to Lemma 1.1 .

As an illustration of Theorem 2.I, consider the inequality

$$
x(t) \leq c+\int_{1}^{t} s^{-3} x(s) d u(s), t \geq 1
$$

where

$$
u(t)=t^{2}+\left(\sum_{k=1}^{k-1} i\right] x_{[k-1, k)}(t), k=2,3, \ldots
$$

Here $x_{A}$, the characteristic function of the set $A$, is defined as $X_{A}(t)=I$ if $t \in A$ and equal to zero otherwise. It is easily seen that $u$ is discontinuous at $t_{k}=k, k=2,3, \ldots ; \lambda_{k}=k$, $\lambda_{k} f\left(t_{k}\right)=k^{-2}<I$ for each $k \geq 2 ; P=\prod_{k=2}^{\infty}\left(I-k^{-2}\right)=\frac{1}{2}$. Estimate 12.4 . gives

$$
x(t) \leq 2 c \cdot \exp \left(2\left(3-t^{-1}\right)\right), t \geq 1
$$

\section{A stability result}

In this section, we obtain sufficient conditions for uniform asymptotic stability of (1.1) in the light of (1.2). It is known [4], that the function $y(t)$ is a solution of (1.1) through $\left(t_{0}, x_{0}\right)$ if and only if it is a solution of the integral equation 


$$
y(t)=x_{0}+\int_{t_{0}}^{t} F(s, y(s)) d s+\int_{t_{0}}^{t} G(s, y(s)) d u(s), \quad t \in J .
$$

Let $F(t, 0) \equiv 0$. We assume that

$\mathrm{H}_{1}$. $\quad F$ satisfies a Lipschitz condition of the type

$$
|F(t, x)-F(t, y)| \leq \alpha(t)|x-y| \text {, for }|x|,|y| \leq a, a>0,
$$

where $\alpha(t)$ is a non-negative function, $d v$-integrable on $J$. Here $v(t)$ is the total variation of $u(t)$ on the interval $\left[t_{0}, t\right]$.

$\mathrm{H}_{2}$. There exists $r>0$ such that if $|x| \leq r$, then

$$
|G(t, x)| \leq \beta(t)|x| \text {, for all } t \geq t_{0},
$$

where $\beta(t)$ is a non-negative function, $d v$-integrable on $J$. Further, let $\lambda_{k} \beta\left(t_{k}\right)<1, k=1,2, \ldots$, where $\lambda_{k}=v\left(t_{k}\right)-v\left(t_{k}-\right)$, and let the series $\sum_{k=1}^{\infty} \lambda_{k} \beta\left(t_{k}\right)$ converge absolutely.

THEOREM 3.1. Let the nulz solution of (1.2) be uniformly asymptotically stable. Suppose that

(i) hypotheses $\mathrm{H}_{1}$ and $\mathrm{H}_{2}$ hold,

(ii) the function $v_{1}^{\prime}$ is non-negative and integrable on $J$, where $v=v_{1}+v_{2}$ is the cononical decomposition of $v$,

$$
\text { (iii) } \int_{t_{0}}^{\infty} \beta(s) d v(s)=L<\infty, \int_{t_{0}}^{\infty}\left\{\alpha(s)+\beta(s) v_{1}^{\prime}(s)\right\}=M<\infty \text {. }
$$

Then there exists $\delta>0$ such that if $t^{\prime} \geq t_{0}$ and $\left|x\left(t^{\prime}\right)\right|<\delta$, the solution $y(t)$ of (1.1) satisfies $|y(t)| \rightarrow 0$ as $t \rightarrow \infty$. In particular if $G(t, 0) \equiv 0$, then the null solution of (1.1) is uniformly asymptotically stable.

Proof. Following the notation in [6], the constants associated with (1.2) regarding the stability of the null solution shall be starred whereas those associated with (1.I) shall not be. Without loss of generality, we may assume that $r \leq a \leq \delta^{*}$. Let $\left|x_{0}\right|<r$. Then we have 


$$
\begin{aligned}
|y(t)-x(t)| & \leq \int_{t_{0}}^{t} \alpha(s)|y(s)-x(s)| d s+\int_{t_{0}}^{t} \beta(s)|y(s)| d v(s) \\
& \leq \int_{t_{0}}^{t} \beta(s)|x(s)| d v(s)+\int_{t_{0}}^{t} \alpha(s)|y(s)-x(s)| d s \\
& +\int_{t_{0}}^{t} B(s)|y(s)-x(s)| d v(s) .
\end{aligned}
$$

Now $v(t)$ is a right-continuous function of bounded variation on $J$ and its discontinuities are the same as those of $u(t)$. Invoking Corollary 2.1, we obtain

$$
|y(t)-x(t)| \leq c P^{-1} L M
$$

where $c=\sup _{t \geq t^{\prime} \geq t_{0}}|x(t)|$. Let $0<\varepsilon \leq r$. Choose $\delta=\delta(\varepsilon)=\delta^{*}(\varepsilon / 2)$

so that $0<\delta<\varepsilon$. Let $t^{\prime} \geq t_{0}$ be so large that $c<\delta P(2 L M)^{-1}$

whenever $\left|x_{0}\right|<\delta^{*}$. Then from (3.1), it follows that

$$
\begin{aligned}
|y(t)| & \leq|y(t)-x(t)|+|x(t)| \\
& <\delta / 2+\varepsilon / 2<\varepsilon,
\end{aligned}
$$

whenever $\left|x_{0}\right|<\delta$. Hence, if $G(t, 0) \equiv 0$, we have shown that the null solution of (1.1) is uniformly stable. The rest follows from (3.1) and the fact that $|x(t)| \rightarrow 0$ as $t \rightarrow \infty$.

REMARKS 3.1. In [4, Theorem 2], a result on quasi-equiasymptotic stability of the null solution of (1.1) is proved by using Lyapunov functions, in which a stronger type of stability (namely, exponential asymptotic stability) on the null solution of (1.2) is assumed. Further, it is assumed that $\left|\lambda_{k}\right| \leq \alpha e^{-c\left(t_{k}-t_{0}\right)}, \alpha, c>0$ and $\alpha$ sufficiently small, which means that the jumps ultimately die down to zero. Our conclusions are stronger under considerably weaker conditions. This is perhaps a consequence of Theorem 2.1.

Theorem 2.1 finds many other useful applications. For example, for the special case 


$$
D x=A x+\bar{F}(t, x)+G(t, x) D u,
$$

where $A$ is an $n \times n$ constant matrix and $\bar{F} \in C\left|J \times R^{n}, R^{n}\right|$, Theorem 4.1 in [6] and similar results in $[1,2,7]$ can be generalized. It is also possible to obtain the bounded region for the solutions and the region of attraction for the stable solutions of (1.1). We omit the details (see [5]).

\section{References}

[1] E.A. Барбашнн [E.A. Barbashin], "Dб устойчивостн по отношению $\mathrm{H}$ импульсным воэдействия" [On stability with respect to impulsive perturbations], Differencial'nye Uravnenija 2 (1966), 863-871; English translation: Differential Equations 2 (1966), 449-453.

[2] Fred Braver, "Nonlinear differential equations with forcing terms", Proc. Amer. Math. Soc. 15 (1964), 758-765.

[3] W.A. Coppel, Stability and asymptotic behavior of differential equations (D.C. Heath, Boston, 1965).

[4] P.C. Das and R.R. Sharma, "Existence and stability of measure differential equations", Czechoslovak Math. J. 22 (97) (1972), 145-158.

[5] Li Yue-Sheng, "The bound, stability and error estimates for the solution of nonlinear differential equations", Chinese Math. Acta $3(1963), 34-41$.

[6] V. Raghavendra and M. Rama Mohana Rao, "On the stability of differential systems with respect to impulsive perturbations", $J$. Math. Anal. App Z. 48 (1974), 515-526.

[7] Aaron Strauss and James A. Yorke, "Perturbation theorems of ordinary differential equations", J. Differential Equations 3 (1967), 15-30.

Department of Mathematics,

Centre for Post-graduate Instruction and Research, University of Bombay,

Panaji, Goa, India. 